\title{
THE FOOT EXPERIMENT: FRAGMENTATION MEASUREMENTS IN PARTICLE THERAPY*
}

\begin{abstract}
A. Alexandrovi,2,3,4, L. Alunni Solestizi5,6, G. Ambrosi5, S. Argirò ${ }^{7,8}$, R. Arteche Diaz'9, N. Bartosik7, G. Battistoni'10, N. Belcari ${ }^{11,12}$, E. Bellinzonaa ${ }^{13}$, S. Bianucci'11, S. Biondi ${ }^{14,15}$, M. G. Bisogni ${ }^{11,12}$, G. Bruni ${ }^{14}$, N. Camarlinghi ${ }^{11,12}$, P. Carra ${ }^{11,12}$, P. Cerello7, E. Ciarrocchi ${ }^{11,12}$, A. Clozza' ${ }^{16}$, S. Colombi 13,17, G. De Lellis',2, A. Del Guerra'11,12, M. De Simoni18,19,

A. Di Crescenzo ${ }^{2,1}$, M. Donetti ${ }^{7,20}$, Y. Dong ${ }^{10,21}$, M. Durante ${ }^{22,23}$, A. Embriaco ${ }^{10}$, M. Emde ${ }^{24}$, R. Faccini ${ }^{18,19}$, V. Ferrero ${ }^{7,8}$, F. Ferroni ${ }^{18,19}$, E. Fiandrini5,6, C. Finck ${ }^{25}$, E. Fiorina ${ }^{11,20}$, M. Fischetti ${ }^{18,26}$, M. Francesconi ${ }^{11,12}$, M. Franchini ${ }^{14,15}$, G. Galati ${ }^{1}$, L. Galli ${ }^{11}$, M. Garbini14,15,27, V. Gentile ${ }^{28}$, G. Giraudo7, R. Hetzel24, E. Iarocci16, M. Ionica5, K. Kanxheri5, A. C. Kraan11, V. Lante' ${ }^{20}$, A. Lauria ${ }^{1,2}$, C. La Tessa ${ }^{13,17}$, E. Lopez Torres7,9, M. Marafini' ${ }^{7,18}$, I. Mattei ${ }^{10}$,

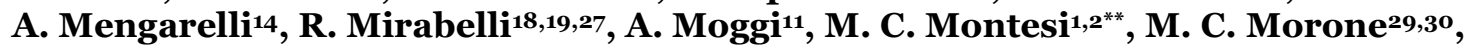
M. Morrocchi'11,12, S. Muraro' ${ }^{11}$, L. Narici'29,30, A. Pastore ${ }^{31}$, N. Pastrone', V. Patera ${ }^{18,26,27}$, F. Pennazio7, P. Placidi5,32, M. Pullia ${ }^{20}$, F. Raffaeli' ${ }^{11}$, L. Ramello ${ }^{7,33}$, R. Ridolfiti5, V. Rosso ${ }^{11,12}$, M. Rovituso'13, C. Sanelli'16, A. Sarti'26,16,27, G. Sartorelli14,15, O. Sato34, S. Savazzi'2o, L. Scavarda ${ }^{7,8}$, A. Schiavi18,26, C. Schuy ${ }^{22}$, E. Scifoni ${ }^{13}$, A. Sciubba ${ }^{18,26,27}$, A. Sécher ${ }^{25}$, M. Selvi'14, L. Servoli5, G. Silvestre5,6, M. Sitta7,33, R. Spighi'14, E. Spiriti'16, G. Sportelli11,12, A. Stahl'24, V. Tioukov ${ }^{1}$, S. Tommasini' ${ }^{16}$, F. Tommasino ${ }^{13,17}$, G. Traini ${ }^{18,19,27}$, S. M. Valle10,21, M. Vanstalle'25, M. Villa'14,15, U. Weber ${ }^{22}$, A. Zoccoli ${ }^{14,15}$
\end{abstract}

'Istituto Nazionale di Fisica Nucleare (INFN), Section of Napoli, Napoli, Italy; ${ }^{2}$ University of Napoli, Department of Physics "E. Pancini", Napoli, Italy; ${ }^{3}$ National University of Science and Technology MISIS, RUS-119049; ${ }^{4}$ Lebedev Physical Institute of the Russian Academy of Sciences, RUS-119991 Moscow, Russia; 5Istituto Nazionale di Fisica Nucleare (INFN), Section of Perugia, Perugia, Italy; ${ }^{6}$ University of Perugia, Department of Physics and Geology, Perugia, Italy; 7Istituto Nazionale di Fisica Nucleare (INFN), Section of Torino, Torino, Italy; ${ }^{8}$ University of Torino, Department of Physics, Torino, Italy; ${ }^{9} \mathrm{CEADEN}$, Centro de Aplicaciones Tecnologicas y Desarrollo Nuclear, Havana, Cuba; ${ }^{10}$ Istituto Nazionale di Fisica Nucleare (INFN), Section of Milano, Milano, Italy; ${ }^{11}$ Istituto Nazionale di Fisica Nucleare (INFN), Section of Pisa, Pisa, Italy; ${ }^{12}$ University of Pisa, Department of Physics, Pisa, Italy; ${ }^{13}$ Trento Institute for Fundamental Physics and Applications, Istituto Nazionale di Fisica Nucleare (TIFPA-INFN), Trento, Italy;

${ }^{14}$ Istituto Nazionale di Fisica Nucleare (INFN), Section of Bologna, Bologna, Italy; ${ }^{15}$ Università of Bologna,

Department of Physics and Astronomy, Bologna, Italy; ${ }^{16}$ Istituto Nazionale di Fisica Nucleare (INFN), Laboratori Nazionali di Frascati, Frascati, Italy; ${ }^{17}$ University of Trento, Department of Physics, Trento, Italy; ${ }^{18}$ Istituto Nazionale di Fisica Nucleare (INFN), Section of Roma 1, Roma, Italy; ${ }^{19}$ University of Roma La Sapienza, Department of Physics, Roma, Italy; ${ }^{\circ}$ Centro Nazionale di Adroterapia Oncologica (CNAO), Pavia, Italy; ${ }^{21}$ Università of Milano, Department of Physics, Milano, Italy; ${ }^{22}$ Biophysics Department, GSI Helmholtzzentrum für Schwerionenforschung, Darmstadt, Germany; ${ }^{23}$ Technische Universität Darmstadt Institut für Festkörperphysik, Darmstadt, Germany; ${ }^{24 R W T H ~ A a c h e n ~}$ University, Physics Institute III B, Aachen, Germany; ${ }^{25 U n i v e r s i t e ̀ ~ d e ~ S t r a s b o u r g, ~ C N R S, ~ I P H C ~ U M R ~ 7871, ~ F-67000 ~}$ Strasbourg, France; ${ }^{26}$ University of Roma La Sapienza, Department of Scienze di Base e Applicate per l'Ingegneria (SBAI), Roma, Italy; ${ }^{27}$ Museo Storico della Fisica e Centro Studi e Ricerche Enrico Fermi, Roma; ${ }^{28}$ Gran Sasso Science Institute, L'Aquila, Italy; ${ }^{29}$ Istituto Nazionale di Fisica Nucleare (INFN), Section of Roma Tor Vergata, Roma, Italy; 30University of Roma Tor Vergata, Department of Physics, Roma, Italy; ${ }^{11}$ Istituto Nazionale di Fisica Nucleare

(INFN), Section of Bari, Bari, Italy; ${ }^{2}$ University of Perugia, Department of Engineering, Perugia, Italy; 33 University of Piemonte Orientale, Department of Science and Technological Innovation, Alessandria, Italy; ${ }^{44 N a g o y a ~ U n i v e r s i t y, ~}$ Department of Physics, Nagoya, Japan

\begin{abstract}
Charged Particle Therapy (CPT) is a powerful radiotherapy technique for the treatment of deep-seated tumours characterized by a large dose released in the Bragg peak area (corresponding to the tumour region) and a small dose delivered to the surrounding healthy tissues. The precise measurement of the fragments produced in the nuclear interactions of charged particle beams with patient tissues is a crucial task to improve the clinical treatment plans. The FOOT (FragmentatiOn Of Target) experiment is an international project, funded by the Istituto Nazionale di Fisica Nucleare (INFN), aimed to study the dose released by the tissues and particle beams fragmentation. The target
\end{abstract}

\footnotetext{
This paper was presented at the Sixth International Conference on Radiation and Applications in Various Fields of Research (RAD 2018), Ohrid, Macedonia, 2018.

montesi@na.infn.it
} 
$\left({ }^{16} \mathrm{O},{ }^{12} \mathrm{C}\right)$ fragmentation induced by $150-400 \mathrm{MeV} / \mathrm{n}$ proton beams will be studied via the inverse kinematic approach, where ${ }^{16} \mathrm{O}$ and ${ }^{12} \mathrm{C}$ therapeutic beams collide on graphite and hydrocarbon target to provide the cross section on Hydrogen. A table-top detector is being developed and it includes a drift chamber as a beam monitor upstream of the target to measure the beam direction, a magnetic spectrometer based on silicon pixel and strip detectors, a scintillating crystal calorimeter able to stop the heavier produced fragments, and a $\triangle E$ detector, with TOF capability, for the particle identification. A setup based on the concept of the "Emulsion Cloud Chamber", coupled with the interaction region of the electronic FOOT setup, will complement the physics program by measuring lighter charged fragments to extend the angular acceptance up to about 70 degrees. In this work, the experimental design and the requirements of the FOOT experiment will be discussed and preliminary results on the emulsion spectrometer tests will be presented.

Key words: Charge particle therapy, fragmentation, instrumentation, nuclear emulsions

\section{INTRODUCTION}

The Charged Particle Therapy (CPT) is a clinical practice that has been expanding to the treatment of deep-seated solid tumours [1]. It is essentially based on the use of protons or carbon ions whose depth-dose profile is characterized by a low amount of the released dose in the entrance channel and by a maximum of the dose deposited in a narrow region, the Bragg Peak (BP), overlapping the cancer volume. This physical behaviour of the dose deposition is essential for sparing healthy tissues but the secondary fragments produced by the nuclear interactions occurring between the ion beam and the patient tissue can release their energy outside the dose profile defined by the beam trajectory. The Relative Biological Effectiveness (RBE), ratio of photon to charged particle dose producing the same biological effect, quantifies the radiation effectiveness and can be affected by the production of secondary fragments. The clinical practice with protons assigns a constant RBE value equal to 1.1, but radiobiological measurements show an increase in RBE. The hypothesis [2] under investigation is that the increase in the $\mathrm{RBE}$ is due to the target fragmentation that generates a spectrum of low energy heavy particles having a range of the order of $10 \div 100 \mu \mathrm{m}$. Experimental data on the differential cross section of produced secondary particles are still missing. Some experiments studied projectile fragmentation for ${ }^{12} \mathrm{C}$ ions, but only for few energies $[3,4]$.

The FOOT (FragmentatiOn Of Target) experiment, funded by Istituto Nazionale di Fisica Nucleare (INFN, Italy), aims to overcome the lack of experimental data in the differential cross section for the production of secondary fragments due the proton and ion beams.

\section{THE FOOT EXPERIMENT}

The aim of the FOOT experiment is to measure the fragment cross section with a maximum uncertainty of $5 \%$ and of the fragment energy spectra with an energy resolution of the order of $1-2 \mathrm{MeV} / \mathrm{n}$ in the inverse kinematic approach. The goals of the experiment are also to achieve the charge and isotopic identification of the fragments with an accuracy of $2-3 \%$ and $5 \%$, respectively.

In proton therapy, the measurement of the target fragmentation is not an easy task due to the low range of the produced fragments (a few tens of microns). To overcome this difficulty, the FOOT project will apply an inverse kinematic approach: instead of studying beams of protons impinging on a target with the composition equivalent to human tissue (98\% $\mathrm{C}, \mathrm{O}$ and $\mathrm{H}$ nuclei), beams of ions $\left({ }^{12} \mathrm{C},{ }^{16} \mathrm{O}\right)$ of the same energy per nucleon will impinge onto a hydrogen enriched target $\left(\mathrm{C}_{2} \mathrm{H}_{4}\right)$. The subtraction of the measured data by using a pure $\mathrm{C}$ and $\mathrm{C}_{2} \mathrm{H}_{4}$ target [3] will provide the cross section of heavy ions on proton:

$$
\frac{d \sigma}{d E_{\text {kin }}}(H)=\frac{1}{4}\left(\frac{d \sigma}{d E_{\text {kin }}}\left(C_{2} H_{4}\right)-2 \frac{d \sigma}{d E_{\text {kin }}}(C)\right)
$$

To this aim, we will perform two beam exposures for each ion type: the first one will be done with ${ }^{12} \mathrm{C}$ ( ${ }^{16}{ }^{16} \mathrm{O}$ ) on the $\mathrm{C}$ target, the second one will be done with ${ }^{12} \mathrm{C}$ (or ${ }^{16} \mathrm{O}$ ) on the $\mathrm{C}_{2} \mathrm{H}_{4}$ target. From these measurements, we will obtain the fragments cross section of ${ }^{12} \mathrm{C}\left({ }^{16} \mathrm{O}\right)$ on the $\mathrm{C}$ and $\mathrm{C}_{2} \mathrm{H}_{4}$ target, respectively.

\section{Yield differential for $\mathrm{T}>30.0 \mathrm{MeV} / \mathrm{n}$}
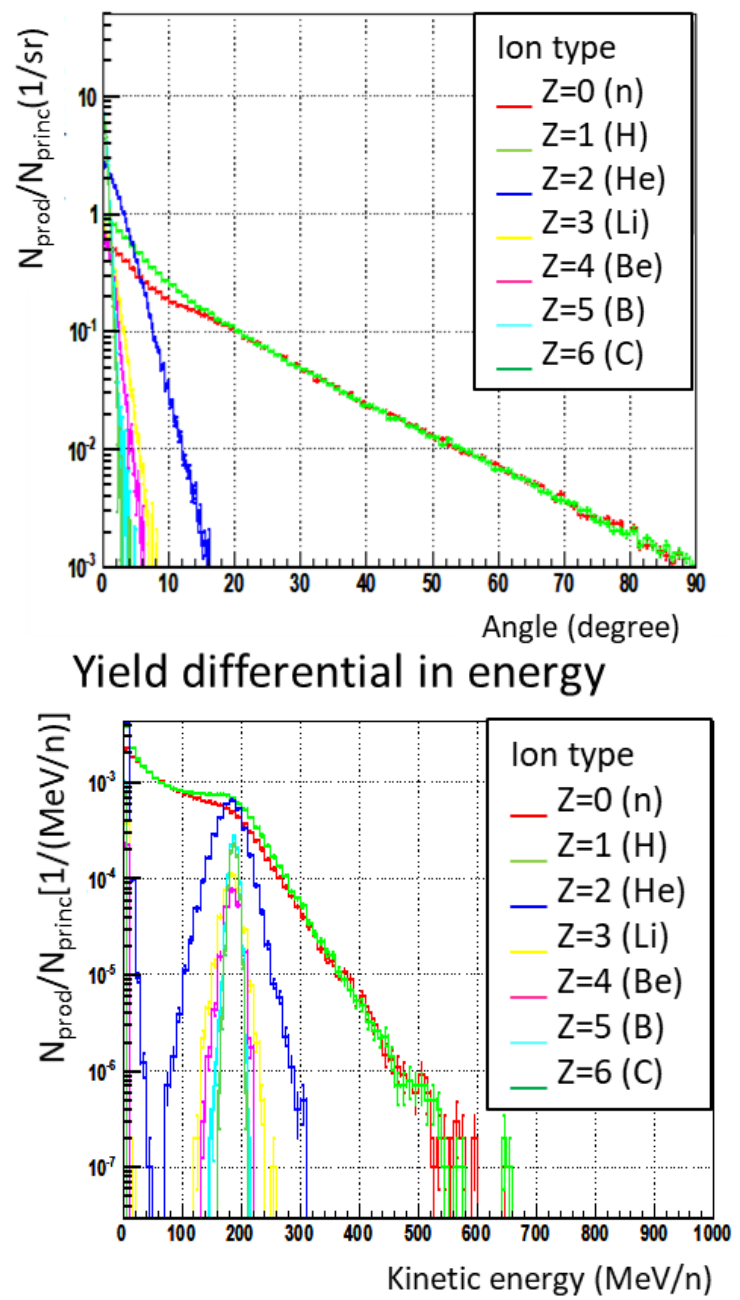

Figure 1. Angular distribution (up) and kinetic energy distribution (down) of the fragments produced by a ${ }^{12} \mathrm{C}$ beam (200 MeV/n) on the $\mathrm{C}$ target (FLUKA 2011). 
The detector design has been guided by the Montecarlo simulation, based on the FLUKA code $[5,6]$. It shows that the light $(Z<3)$ fragments have a wide emission angle (up to $90^{\circ}$ ) while the heavier fragments are forward peaked within a polar angle of about $10^{\circ}$. In Figure 1, the simulated angular (up) and energy distribution per nucleon (down) produced by a ${ }^{12} \mathrm{C}(200$ $\mathrm{MeV}$ ) beam on the $\mathrm{C}$ target is shown.

Taking into account these results and the need to realise a "portable" experimental setup fitting the space limitations of the different experimental and treatment rooms, the FOOT experiment consists of two complementary setups:

1. an electronic setup based on a magnetic spectrometer dedicated to the momentum reconstruction and an identification section made of a plastic scintillator and a calorimeter. This setup aims to measure fragments heavier than ${ }^{4} \mathrm{He}(\mathrm{Z} \geq 3)$ and covering a polar angle of $\pm 10^{\circ}$ with respect to the beam axis;

2. a setup based on an emulsion spectrometer supplying complementary measurements for light fragments emitted at an angle up to about $70^{\circ}$.

The FOOT data taking will take place using $4 \mathrm{He},{ }^{12} \mathrm{C}$ and ${ }^{16} \mathrm{O}$ beams at energies ranging from $150 \mathrm{MeV} / \mathrm{n}$ to 400 $\mathrm{MeV} / \mathrm{n}$ and have a great interest for clinical applications. Other applications, as radioprotection in space, are interested in the knowledge of fragmentation processes required for the design of spacecraft shielding dedicated to long duration space missions. Target fragmentation studies using a ${ }^{4} \mathrm{He}$ beam with energy reaching $700 \mathrm{MeV} / \mathrm{n}$ are also programmed.

\section{THE ELECTRONIC DETECTOR SETUP}

The electronic detector setup measures the following quantities related to the produced fragments: momentum (p), kinetic energy $\left(\mathrm{E}_{\mathrm{k}}\right)$, Time Of Flight (TOF) and fragments energy loss $(d E / d x)$. The detector geometry consists of three main regions: the upstream/target region, the magnetic tracking region and the identification region. Figure 2 shows a schematic view of the detector and a brief description of different subsections is reported below.

1. The upstream/target region: the plastic scintillator counter $(250 \mu \mathrm{m}$ thick) provides the start signal of the TOF and the trigger system, downstream a drift chamber operates as a beam monitor tracking the direction and the position of the beam. The target is positioned beyond the beam monitor.

2. The magnetic region: it is composed of three stations of pixel and strip detectors allocated between and downstream of the magnets providing the vertex reconstruction and the initial tracking of the produced fragments; two permanent dipole magnets supply the magnetic field (up to $1.4 \mathrm{~T}$ ) in the Halbach geometry [7] that allows to contain the overall weight of the detector.

3. The identification region: it is the distal part of the detector located at about $1 \mathrm{~m}$ away from the target. It is composed of two orthogonal planes of plastic scintillator rods, providing the stop of the TOF and the measurement of the energy loss, and of a BGO calorimeter for the fragment kinetic energy measurements.

The TOF, measured by the start counter and the scintillator, has an estimated resolution at the level of 70-100 ps. The silicon detectors, in the magnetic region, are able to perform momentum measurements with an expected resolution $(\sigma(p) / p)$ at the level of $5 \%$, while the resolution supplied by the scintillator and by the calorimeter is at the level of $3 \%$ and $2 \%$ respectively in the measurement of the energy loss $(\sigma(\Delta E) /(\Delta E))$ and in the kinetic energy $\left(\sigma\left(E_{k}\right) /\left(E_{k}\right)\right)$.

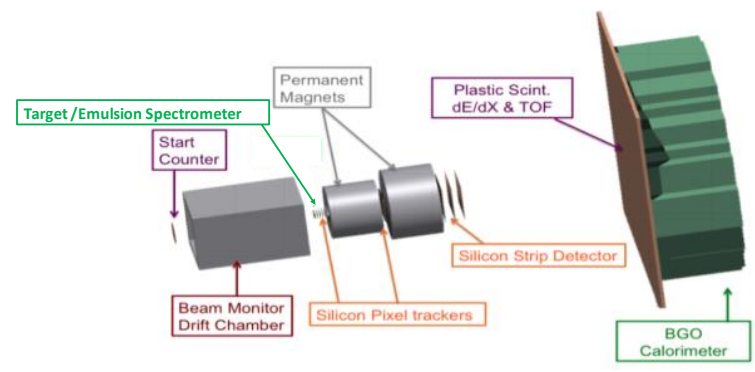

Figure 2. Schematic view of the FOOT detector. The total detector length is about $2 \mathrm{~m}$ and the maximum transversal area is $50 \times 50 \mathrm{~cm}^{2}$.

\subsection{Electronic detector performances}

The simulation studies have been carried out to characterize the events occurring in the FOOT electronic detector. The performances have been obtained by analyzing the events coming from the interaction of an ${ }^{16} \mathrm{O}$ beam $(200 \mathrm{MeV} / \mathrm{n})$ with a $\mathrm{C}_{2} \mathrm{H}_{4}$ target.

The differential cross section measurement of the produced fragments is a task that can be achieved through the accurate determination of fragments atomic $(\mathrm{Z})$ and mass number (A). The analysis has been focused on the study of produced fragments with $\mathrm{Z}$ number ranging from 1 up to 8 . All the quantities necessary for the $\mathrm{Z}$ and $\mathrm{A}$ fragments identification derive from the measurements of the TOF, the momentum, the energy release $(\Delta E)$ and the kinetic energy.

The TOF resolution ranges from 70 to 100 ps varying from ${ }^{12} \mathrm{C}$ to Hydrogen, as measured in a dedicated test beam. The $\beta(=\mathrm{v} / \mathrm{c})$ of the fragment is derived from the TOF and the trajectory length of the track.

The momentum reconstruction has been obtained by applying a Gaussian smearing of $5 \%$, independently of the momentum and of the types of fragments.

The energy release, $\Delta E$, has been reconstructed by the energy deposited in the plastic scintillator. The $\Delta \mathrm{E}$ resolution ranges from $3 \%$ to $10 \%$ as a function of the deposited energy and it has been obtained in a dedicated test beam.

The kinetic energy $E_{k}$ has been reconstructed by adding the energy deposition in the plastic scintillator and in the calorimeter. The resolution on the deposited energy in the calorimeter has been obtained by a fixed Gaussian smearing of $1.5 \%$ independent on the energy deposition.

The resolution on the A identification is strongly dependent both on the energy loss by fragments due to 
ionization processes before reaching the calorimeter and on the leakage inside it, mostly generated by neutrons emission. It is necessary to have a redundant detector capable of reconstructing and identifying those events where the final state has undergone interaction before reaching the calorimeter or when a part of the energy escaped the apparatus due to neutron emission.

Only the tracks travelling through the detector are considered to evaluate the detector performances. The plastic scintillator is dedicated to the $\mathrm{Z}$ determination by measuring the energy loss and the TOF for the $\beta$ evaluation. The $\mathrm{Z}$ reconstruction is presented in Figure 3 for all the studied fragments; the peaks are well distinguished from each other, allowing a clear charge identification. The $\mathrm{Z}$ resolution improves with increasing the charge of the fragments ranging from $2 \%$ for ${ }^{16} \mathrm{O}$ to $6 \%$ for ${ }^{1} \mathrm{H}$.

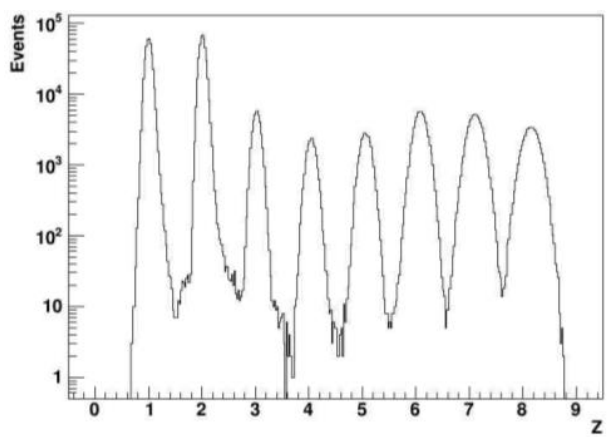

Figure 3. Reconstruction of charge number $\mathrm{Z}$ for all fragments crossing the detector.

The mass number A can be estimated by combining the kinematic determinations supplied by different FOOT sub-detectors in different ways. For example, the simultaneous determination of $\beta$, $\mathrm{p}$ and $\mathrm{E}_{\mathrm{k}}$, supplied by the TOF, the tracking in magnetic field and the calorimeter detectors respectively, allows the estimation of the mass number according to the following formulae:

$$
A_{1}=\frac{p}{U \beta \gamma} \quad A_{2}=\frac{E_{k}}{U(\gamma-1)} \quad A_{3}=\frac{p^{2}-E_{k}^{2}}{2 \mathrm{U} E_{k}}
$$

where $U$ is the Unified Atomic mass $(931.5 \mathrm{MeV})$ and $\gamma$ $=1 /\left(1-\beta^{2}\right)^{1 / 2}$.

The three A determinations have a correlation due to the presence of a common subdetector in each couple of A definitions. The best determination of $\mathrm{A}$ is through two different fit procedures:

1. a standard $\chi^{2}$ approach;

2. an Augmented Lagrangian Method (ALM) approach [8].

The results on the mass number determination, for example for ${ }^{12} \mathrm{C}$, obtained by the standard $\chi^{2}$ and ALM fit are presented in Figure 4.

The two methods give similar results: the peak position is centered on the expected value and the resolution is about $4 \%$, similar to that obtained with the direct measurement of $\mathrm{A}$. The visible tails, showing bad reconstructed number of mass, can be suppressed optimizing the $\chi^{2}$ cut.
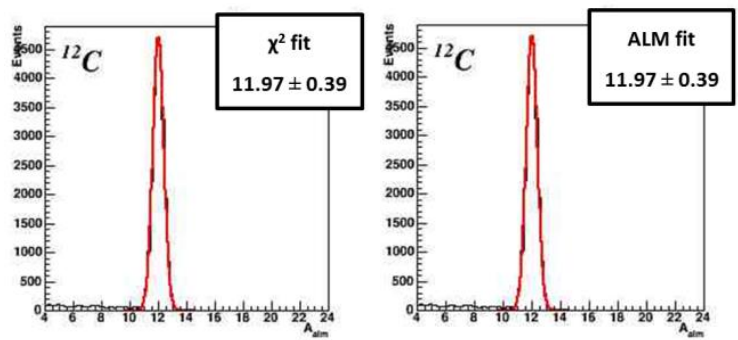

Figure 4. Determination of the mass A (for ${ }^{12} \mathrm{C}$ ) with the standard $\chi^{2}$ method fit (left) and with the ALM fit (right).

\section{THE EMULSION SPECTROMETER SETUP}

The emulsion spectrometer is meant to detect light $(Z \leq 3)$ fragments emitted at a large angle (up to about $70^{\circ}$ ). The Emulsion Cloud Chamber (ECC) technology, alternating nuclear emulsion films with passive material, is the basis of the emulsion spectrometer [912]. The ECC acts as a micrometric tracking device, ionization chamber and a spectrometer for isotope separation.

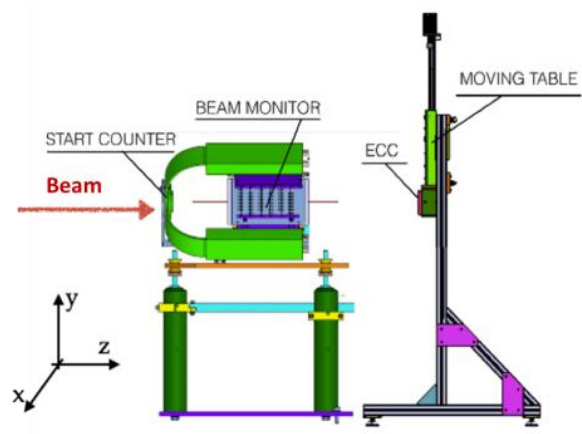

Figure 5. Emulsion spectrometer exposure geometry: the ECC is hosted on a XY moving stage and positioned downstream with respect the start counter and the beam monitor.

The emulsion spectrometer setup will include the start counter and the beam monitor of the electronic setup (see Figure 5) in order to monitor the integrated particle density in the nuclear emulsion. These detectors will provide a feedback signal to control the movement of the XY stage hosting the ECC during the beam spill to avoid the piling up of different interactions.

The nuclear emulsion films consist of two $50 \mu \mathrm{m}$ thick sensitive emulsion layers deposited on both sides of a plastic base, $200 \mu \mathrm{m}$ thick, resulting in a total thickness of $300 \mu \mathrm{m}$. AgBr crystals, dispersed in a gelatine matrix, are sensitive to ionizing particles and record their trajectory acting as latent image centres. A chemical process of development produces the latent image as silver grains recorded as dark pixels by an optical microscope. A dedicated software recognizes aligned clusters of dark pixels producing a "base-track" related to the path of the charged particle (see Figure 6) [13]. The connection of base-tracks, belonging to different emulsions and defining a straight line, forms volume-tracks. 


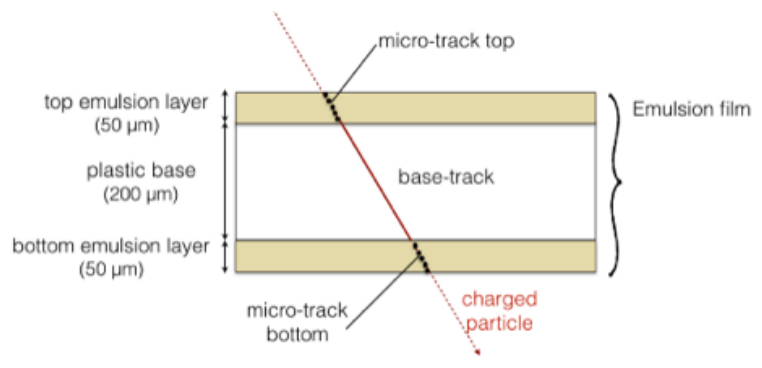

Figure 6. Tracking reconstruction scheme: a sequence of clusters aligned in the top and bottom layers forms a microtrack; geometrically aligned top and bottom micro-tracks form base-tracks.

The emulsion spectrometer is organized in three sections (see Figure 7).

1. Section I (Vertex): it is composed by alternating emulsion films, acting as micrometric trackers, and passive layers of $\mathrm{C}$ or $\mathrm{C}_{2} \mathrm{H}_{4}$ acting as the target where secondary fragments originate.

2. Section II (Charge separation): it consists of emulsion films for charge identification.

3. Section III (Momentum measurements and isotopic identification): it consists of emulsion films alternated with passive materials of increasing density (plastic, steel and lead) to measure the particle range and momentum through the detection of Multiple Coulomb Scattering [14,15].

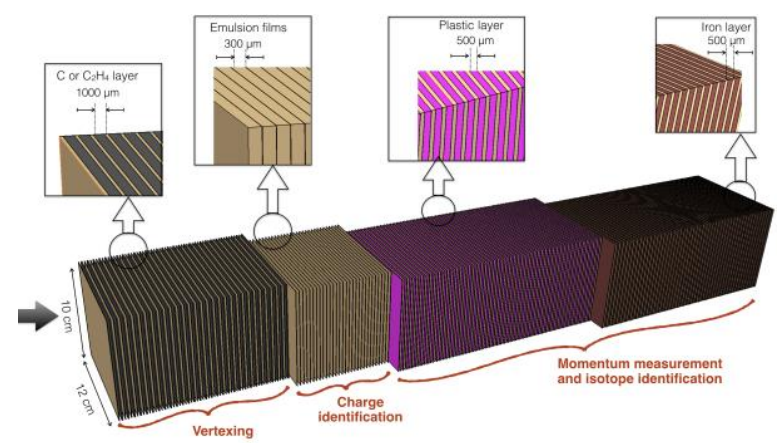

Figure 7. Scheme of the emulsion spectrometer structure for the FOOT experiment: the vertexing section composed by alternating emulsion films and passive layers of $\mathrm{C}$ or $\mathrm{C}_{2} \mathrm{H}_{4}$; the charge identification section consisting in a sequence of emulsion films; the momentum measurement and isotopic identification section consisting in emulsion films alternated with passive materials.

The length of the vertex section is optimized by the conflicting requirements to get a statistically significant number of interactions and avoid absorbing the secondary particles. About $20 \%$ of ${ }^{12} \mathrm{C}$ ions are expected to interact within $30 \mathrm{~mm}$ of $\mathrm{C}$ and consequently the total length of this section could be about $4 \mathrm{~cm}$ ( 31 emulsion layers interleaved with $30 \mathrm{C}$ layers).

The second section is meant to measure the charge of the fragments. In nuclear emulsions, the grain density along the particles trajectory is almost proportional to the particle energy loss over a certain range, above which a saturation effect occurs. By keeping the emulsion at moderate temperatures (above $28^{\circ} \mathrm{C}$ ) and humidity (around 98\%) before the chemical development, the saturation effects can be avoided by a controlled erasing of the grains along the particle trajectory. As a consequence, the emulsions become sensitive to particles with higher ionization. A tuning of the temperature in the refreshing process erases the tracks due to the particles with charge equal to 1 while preserving those induced by particles with higher charge. The implementation of the refreshing technique in the second section foresees the use of elementary cells, each containing three emulsion films. Each film in the cell, denoted as $R_{0}, R_{1}$ and $R_{2}$, undergoes a specific thermal treatment. $R_{0}$ films are not refreshed, while $R_{1}$ and $R_{2}$ ones are kept for a given time interval at $98 \%$ relative humidity and at a temperature ranging from $28^{\circ} \mathrm{C}$ to $36^{\circ} \mathrm{C}$. For each refreshing condition, a track is characterized by three volume variables, i.e. the sum along the track of all the pixels recorded by the CMOS, referred to as VRo, VR1 and VR2, respectively. The charge separation is obtained by the correlations between pairs of volume variables as demonstrated in [10].

In the third section of the emulsion spectrometer, the particle range is measured accounting for its stopping point and slope. The nuclear emulsions register the $x-y$ spatial position and slope $\left(\theta_{\mathrm{x}}, \theta_{\mathrm{y}}\right)$ for each particle track allowing to estimate the particle momentum by the MCS method according the following formula [16]:

$$
\delta \theta=\frac{13.6}{p \beta} \sqrt{\frac{x}{x_{0}}}\left[1+0.038 \ln \left(\frac{x}{x_{0}}\right)\right]
$$

where $p$ is the particle momentum, $\beta$ the particle velocity, $\delta \theta$ the deviation of the track slope along its path, $x$ the traversed distance and $X_{o}$ the passive material radiation length $[15,16]$. A dedicated algorithm to measure the particle momentum through the MCS detection is used. The algorithm will be extended to include the case where the energy loss along the trajectory is not negligible. The total number of passive layers of this section is set according to the energy of the incident beam and it will consist of both lighter (plastic) and heavier (steel, lead) passive materials. The combination of the particle range and momentum measurements will allow the isotopic identification of the fragments.

The success of the application of emulsions in different fields like neutrino physics and nuclear physics applied to medicine relies on the impressive development of fully automated scanning systems. The last generation microscopes have reached the scanning speed of $190 \mathrm{~cm}^{2} / \mathrm{h}$ with wide angular acceptances (more than $70^{\circ}$ ) [17-19].

\subsection{Emulsion spectrometer test}

Preliminary tests have been performed to calibrate the sensitivity of a new batch of nuclear emulsions, produced at Nagoya University, and to test the MCS algorithm for the momentum reconstruction. Two ECC detectors were exposed to proton and deuterium beams of $80 \mathrm{MeV} / \mathrm{n}$ kinetic energy at LNS laboratory (Catania, Italy). The ECC structure developed for this study consists of 21 nuclear emulsion films interleaved with 20 steel plates $\left(0.5 \mathrm{~mm}\right.$ thick, $\left.7.97 \mathrm{~g} / \mathrm{cm}^{3}\right)$. The ECC dimensions are $4.0 \times 5.0 \mathrm{~cm}^{2}$ in the plane transverse to the beam and $1.63 \mathrm{~cm}$ longitudinally. The ECC structure 
was packed under vacuum with a $100 \mu \mathrm{m}$ thick lighttight aluminium envelope. The beam exposure time was calibrated in order to integrate a few tens of particles $/ \mathrm{mm}^{2}$ impinging on the nuclear emulsion.

After the film chemical development, the emulsions were analysed with automated microscopes and the volume particle tracks were reconstructed by using a dedicated software aimed to find and fit base-tracks geometrically aligned along the ECC structure. For each volume track, we measured the $x-y$ spatial coordinates with micrometric accuracy and the slope with a resolution of a few mrad.

We estimate the particle momentum by the MCS method by evaluating the angular deviation between the base-track and the volume track in each nuclear emulsion. Then, by reversing the formula (3), the $1 / \mathrm{p} \beta$ quantity is computed. The $1 / \mathrm{p} \beta$ distributions for proton and deuterium are reported in Figure 8 . The $1 / \mathrm{p} \beta$ mean value obtained by the Gaussian fit is in agreement with the expected one, calculated starting from the energy beam $(80 \mathrm{MeV} / \mathrm{n})$ (see Table 1$)$. These results show the isotopic identification with nuclear emulsions of different beams and the potentiality for the isotopic identification of an event by event basis.

Table 1 . Expected and measured values for $1 / \mathrm{p} \beta$ quantity obtained for proton and deuterium exposure.

\begin{tabular}{|c|c|c|}
\hline & $\mathrm{H}$ & $\mathrm{D}$ \\
\hline $1 / \mathrm{p} \beta_{\text {expected }}\left((\mathrm{GeV} / \mathrm{c})^{-1}\right)$ & 6.8 & 3.4 \\
\hline $1 / \mathrm{p} \beta$ measured $\left((\mathrm{GeV} / \mathrm{c})^{-1}\right)$ & $8.9 \pm 2.9$ & $3.9 \pm 1.3$ \\
\hline
\end{tabular}

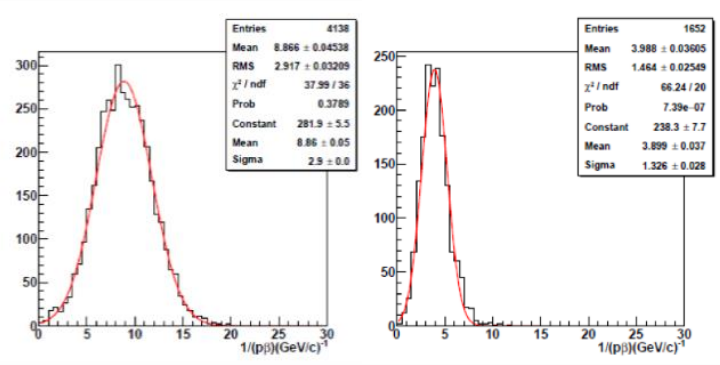

Figure 8 . Distribution of $1 / \mathrm{p} \beta$ obtained for a proton beam (left) and for a deuterium beam (right) at $80 \mathrm{MeV}$. The Gaussian fit is reported in red line and the fit parameters are in Table 1.

\section{CONCLUSION}

In the framework of the FOOT collaboration, a detector based on a double experimental setup is under design and construction. The aim of the FOOT detector is to study:

1. target fragmentation in proton therapy;

2. projectile fragmentation in ion therapy and space radioprotection.

The data taking will take place by using $4 \mathrm{He},{ }^{12} \mathrm{C}$ and ${ }^{16} \mathrm{O}$ beams in the $150-700 \mathrm{MeV} / \mathrm{n}$ energy range. Experimental sites will be the CNAO center (Pavia, Italy), the HIT center (Heidelberg, Germany) and the GSI (Darmstadt, Germany). Some preliminary tests on calorimeter, scintillator, silicon and emulsion detectors have been performed at the CNAO center, at the LNS
(Catania, Italy) and at the Proton Therapy Center (Trento, Italy) and the results are under analysis.

The first data taking with emulsion setup is scheduled in November 2018 at the GSI by using ${ }^{4} \mathrm{He}$ beam $(700 \mathrm{MeV} / \mathrm{n})$ on a $\mathrm{C}$ and $\mathrm{C}_{2} \mathrm{H}_{4}$ target for space radioprotection studies.

Acknowledgements: The R\&D of the FOOT experiment has been funded by INFN, with the contribution of the Centro Fermi Institute, for 2017. INFN funded the FOOT experiment for the 2018-2021 period.

\section{REFERENCES}

1. M. Durante, J. S. Loeffler, "Charged Particles in Radiation Oncology," Nature Rev. Clin. Oncol., vol. 7, pp. 37 - 43, Jan. 2010

DOI: 10.1038/nrclinonc.2009.183

PMid: 19949433

2. F. Tommasino, M. Durante, "Proton Radiobiology," Cancers, vol. 7, pp. $353-381$, Mar. 2015.

DOI: $10.3390 /$ cancers7010353 PMid: 25686476

3. J. Dudouet et al., "Double Differential Fragmentation Cross-Section Measurements of $95 \mathrm{MeV} / \mathrm{n}{ }^{12} \mathrm{C}$ Beams on Thin Targets for Hadron Therapy," Phys. Rev. C, vol. 88, no. 2, 024606, Aug. 2013.

DOI: 10.1103/PhysRevC.88.024606

4. M. Toppi et al., "Measurements of Fragmentation Cross Section of ${ }^{12} \mathrm{C}$ ions on a thin Gold target with the FIRST Apparatus," Phys. Rev. C, vol. 93, 064601, Jun. 2016. DOI: 10.1103/PhysRevC.93.064601

5. A. Ferrari, P. Sala, A. Fassò, J. Ranft, FLUKA: A MultiParticle Transport Code, Rep. CERN-2005-10, INFN/TC 05/11，SLAC-R-773，CERN，INFN，SLAC, Geneva, Switzerland, 2005

Retrieved from: https://www.slac.stanford.edu/pubs/sl acreports/reports16/slac-r-773.pdf; Retieved on: Jun. 2, 2018

6. T. T. Boehlen et al., "The FLUKA code: developments and challenges for high energy and medical applications," Nucl. Data Sheets, vol. 120, pp. $211-214$, Jun. 2014. DOI: $10.1016 /$ j.nds.2014.07.049

7. K. Halbach, "Design of permanent multipole magnets with oriented rare earth cobalt material," Nucl. Instrum. Methods, vol. 169, pp. 1 - 10, Feb. 1980. DOI: 10.1016/0029-554X(80)90094-4

8. W. Sang Cho et al., "OPTIMASS: A Package for the Minimization of Kinematic Mass Functions with Constraints," deposited at arXiv Jan. 12, 2016. arXiv: $1508.00589 \mathrm{v} 2$

9. G. De Lellis et al., "Emulsion Cloud Chamber technique to measure the fragmentation of a high-energy carbon beam," JINST, vol. 2, Po6004, Jun. 2007. DOI:10.1088/1748-0221/2/06/Po6004

10. G. De Lellis et al., "Measurement of the fragmentation of Carbon nuclei used in hadron-therapy," Nucl. Phys. A, vol. 853, no. 1, pp. 124 - 134, Mar. 2011. DOI: 10.1016/j.nuclphysa.2011.01.019

11. A. Alexandrov et al., "Measurement of large angle fragments induced by $400 \mathrm{MeV} \mathrm{n}^{-1}$ carbon ion beams," Meas. Sci. Technol., vol. 26, no. 9, 094001, Sep. 2015. DOI: 10.1088/0957-0233/26/9/094001

12. A. Alexandrov et al., "Measurement of ${ }^{12} \mathrm{C}$ ions beam fragmentation at large angle with an emulsion cloud chamber," JINST, vol. 12, Po8013, Aug. 2017. DOI: 10.1088/1748-0221/12/08/Po8013 
13. L. Arrabito et al., "Track reconstruction in the emulsionlead target of the OPERA experiment using the ESS microscope," JINST, vol. 2, Po5004, May 2007.

DOI: $10.1088 / 1748-0221 / 2 / 05 / P 05004$

14. G. De Lellis et al., "Momentum measurement by the angular method in the Emulsion Cloud Chamber," Nucl. Instrum. Methods Phys. Res. A, vol. 512, no. 3, pp. $539-545$, Oct. 2003.

DOI: 10.1016/So168-9002(03)02016-3

15. N. Agafonova et al., "Momentum measurement by the Multiple Coulomb Scattering method in the OPERA lead emulsion target," New J. Phys., vol. 14, 013026 , Jan. 2012. DOI: $10.1088 / 1367-2630 / 14 / 1 / 013026$

16. M. Tanabashi et al. "Reviev of Particle Physics," Phys. Rev. D, vol. 98, O30001, Aug. 2018
DOI: 10.1103/PhysRevD.98.030001

17. A. Alexandrov et al., "A new fast scanning system for the measurement of large angle tracks in nuclear emulsions," JINST, vol. 10, P11006, Nov. 2015

DOI: $10.1088 / 1748-0221 / 10 / 11 /$ P11006

18. A. Alexandrov et al., "A new generation scanning system for the high-speed analysis of nuclear emulsions," JINST, vol. 11, Po6002, Jun. 2016. DOI: $10.1088 / 1748-0221 / 11 / 06 /$ Po6oo2

19. A. Alexandrov et al., "The continuous motion technique for the new generation scanning system," Sci. Rep., vol. 7, 7310, Aug. 2017.

DOI: $10.1038 / \mathrm{s} 41598-017-07869-3$ 